\title{
Fatores que Influenciam os Resultados do Tratamento Não-Medicamentoso das Mastalgias Cíclicas
}

\author{
Factors that Influence the Outcome of the Nonpharmacological \\ Treatment of Cyclical Mastalgias
}

Flávio Nunes Sivini ${ }^{1}$, Aurélio Molina ${ }^{1}$, Cícero Ferreira Fernandes Costa ${ }^{1}$, Flávio Murilo Pinto Sivini ${ }^{2}$

\begin{abstract}
RESUM0
Objetivo: avaliar fatores que possam influenciar os resultados do tratamento nãomedicamentoso (orientação verbal) em mulheres com mastalgia cíclica.

Métodos: conduzimos um estudo do tipo experimental não controlado com uma amostra de 128 mulheres com uma história clara de mastalgia cíclica, tratadas com orientação verbal. Uma escala analógica visual da dor foi usada antes e após tratamento, a fim de avaliar a sua gravidade, e classificamos as mastalgias em graus I (leve), II (moderado) e III (intensa), de acordo com a intensidade da dor. Usamos também o Cardiff Breast Score (CBS) modificado para avaliar a resposta clínica ao tratamento. A análise dos dados foi feita com o teste do $\chi^{2}$ (Epi-Info 6.04).

Resultados: verificamos que fatores como paridade, menarca, idade do primeiro parto a termo e amamentação não influenciaram significativamente $(p=0,19, p=0,31, p=0,80$ e $p=0,54$, respectivamente) os resultados do tratamento não-medicamentoso (orientação verbal). Por outro lado, quando levamos em consideração a idade, observamos que 26 (78,8\%) pacientes com 40 anos ou mais de idade foram mais beneficiadas com a orientação verbal, com diferença estatisticamente significante $(p=0,01)$ quando comparadas aos grupos mais jovens.

Conclusão: fatores reprodutivos como paridade, menarca, idade do primeiro parto a termo e amamentação não influenciaram os resultados do tratamento não medicamentoso (orientação verbal). O fator idade, especificamente, influenciou significativamente os resultados.
\end{abstract}

PALAVRAS CHAVE: Mama: doenças benignas. Mastalgia. Orientação Verbal.

\section{Introdução}

A dor é o sintoma mamário mais comum segundo extensa literatura médica internacional. A mastalgia cíclica, particularmente, corresponde a dois terços dos $\operatorname{casos}^{1,2}$.

No que diz respeito à paridade, Mansel $^{3}$ afirma que o curso da mastalgia não é afetado por ela, entretanto, Barros et al. ${ }^{4}$ observaram maior freqüência de mastalgia em nuliparas, sugerindo que

\footnotetext{
${ }^{1}$ Departamento Materno-Infantil da Faculdade de Ciências Médicas da Universidade de Pernambuco.

${ }^{2}$ Instituto de Ciências Biológicas da Faculdade de Ciências Médicas da Universidade de Pernambuco.

Correspondência:

Flávio Nunes Sivini

Av. Boa Viagem, 5600 - apartamento 302

51030-000 - Recife - PE
}

gestações sucessivas tendem a proteger do sintoma. São contraditórios os achados de que a mastalgia cíclica é mais freqüente em nulíparas ou naquelas que tiveram o primeiro filho com tenra idade, porque em uma determinada população, baixa paridade e idade tardia de ter o primeiro filho estão geralmente associados ${ }^{5}$.

No que diz respeito à idade do primeiro parto a termo, Fentiman ${ }^{6}$ afirma que mulheres com dor mamária que não respondem a orientação verbal (reassurance) nem a placebo, como piridoxina (B6), ou a diuréticos, com maior probabilidade tiveram seu primeiro filho muito jovens. Esse autor considera que esse fator de risco é mais sociológico do que biológico, mas geralmente se observam mulheres com o nivel de ansiedade maior do que aquele encontrado na maioria das pacientes. 
Em relação à amamentação, Wetzig ${ }^{2}$ afirma que a sua falta foi identificada como um fator significante na história das pacientes que procuram as clínicas especializadas com queixa de mastalgia.

A orientação verbal como forma de tratamento das mastalgias cíclicas deve ser recomendada sempre como primeira opção terapêutica ${ }^{7-11}$, particularmente levando-se em consideração os seguintes aspectos: existe grande número de opções terapêuticas no tratamento das mastalgias, incluindo a prescrição de diversos fármacos, muitas vezes de custo elevado, alguns com eficácia nem sempre comprovada e outros com efeitos colaterais às vezes significativos. É reconhecido também que metade das pacientes tratadas terá recorrência num período curto de tempo, após terem experimentado melhora e suspendido o tratamento ${ }^{12-14}$.

A orientação verbal consiste na explicação convincente sobre a natureza não-neoplásica do sintoma, a não-elevação do risco relativo para o câncer em suas portadoras e suas possiveis causas, além de sua evolução natural.

Por outro lado, não encontramos, na literatura pesquisada, referência de como fatores como paridade, menarca, idade do primeiro parto a termo, amamentação e idade pudessem, de alguma maneira, interferir na resposta ao tratamento nãomedicamentoso (orientação verbal) das mastalgias cíclicas, levando-nos a um especial interesse no tema.

\section{Pacientes e Métodos}

Foi realizado um trabalho prospectivo do tipo experimental não controlado, com 128 mulheres com queixa inequívoca de mastalgia cíclica que procuraram ou foram encaminhadas ao ambulatório de Patologia Mamária, do Centro Integrado de Saúde Amaury de Medeiros (CISAM) da Faculdade de Ciências Médicas/UPE. Estas foram atendidas no período de 23 de dezembro de 1998 a 26 de agosto de 1999. Foram excluídas do estudo pacientes com doença mamária (nódulos císticos ou sólidos, derrames, etc.) detectável ao exame clínico ou após propedêutica pertinente (mamografia e/ou ultra-sonografia) ou que estivessem fazendo uso de drogas que pudessem de alguma maneira influir na presença ou grau de dor mamária: anticoncepcional hormonal, tamoxifeno, bromocriptina, etc. Foram também excluidas as pacientes com dor referida na mama, mas de causa extramamária e portadoras de mastalgia acíclica.
A mastalgia cíclica (variável dependente) foi classificada em quatro diferentes categorias, levando-se em consideração a intensidade maior ou menor da dor: grau I (leve), grau II (moderado), grau III (intenso) e grau 0 (ausência de dor).

$\mathrm{Na}$ mastalgia grau I (leve), as mamas eram pouco dolorosas, a dor não interferia nas atividades habituais e não requeria analgésicos. $\mathrm{Na}$ mastalgia grau II (moderado), as mamas eram moderadamente dolorosas, a dor chegava a interferir com as atividades habituais e raramente requeria analgésicos. Na mastalgia grau III (intenso), as mamas eram intensamente dolorosas, a dor interferia nas atividades habituais e quase sempre necessitava de analgésicos. No grau 0 de mastalgia (ausência de dor), eram classificadas as pacientes que após tratamento não-medicamentoso (orientação verbal) sentiam-se curadas.

A orientação verbal fundamenta-se na abordagem sistemática dos seguintes aspectos: explicação convincente sobre a natureza não-neoplásica do sintoma; a não-elevação do risco relativo para o câncer nas portadoras de dor mamária; as possiveis causas do sintoma e a sua evolução natural. Tudo isso, após exame físico detalhado com propedêutica pertinente. Nesse tipo de abordagem, a relação médico-paciente é fundamental.

Para permitir um referencial comparativo pré e pós-tratamento, as pacientes submeteramse a uma avaliação basal da dor que consistiu na verificação de parâmetros, de base subjetiva, informados pela paciente com supervisão e orientação do pesquisador.

Empregamos a classificação sintomática da dor mamária em graus I, II ou III (anamnese) (parâmetro básico, alicerce do estudo); atribuição pela própria paciente de nota de 0 a 10 para a intensidade da dor mamária (nota 10 para a dor máxima) (parâmetro secundário, usado como apoio); a avaliação da intensidade da dor mamária mediante o uso da escala linear analógica visual da dor, que foi apresentada à paciente pelo pesquisador) (parâmetro secundário, também usado como apoio).

Para a análise evolutiva do quadro doloroso, foi utilizado o CBS - Cardiff Breast Score modifica$\mathrm{do}^{15}$ (usado unicamente após o tratamento), que consistia na auto-avaliação da paciente em relação à sua sintomatologia dolorosa. Após a autoavaliação a evolução foi categorizada em: cura quando não há qualquer dor residual após tratamento; melhora - quando permanece alguma dor residual, mas considerada pela paciente perfeitamente tolerável; nenhuma resposta - a dor permanece inalterada e piora quando há um agravamento da sintomatologia.

A eficácia do tratamento não-medicamen- 
toso foi constatada quando pelo menos três dos quatro parâmetros (classificação sintomática da dor, nota atribuída pela paciente, escala linear analógica visual da dor e análise evolutiva do quadro doloroso) utilizados com essa finalidade mostrassem diminuição de intensidade da dor em qualquer nível.

Em relação aos aspectos éticos, o estudo foi submetido ao Comitê de Ética em pesquisa do CISAM e aprovado.

\section{Resultados}

A média de idade das pacientes com mastalgia cíclica foi de 34 anos (variação entre 20 e 51 anos). As mastalgias cíclicas de graus II e III foram mais prevalentes entre as idades de 20 e 29 anos $(77,1 \%)$ quando comparadas com os outros grupos etários $(55,2 \%$ e $60,7 \%$ respectivamente) e essa diferença foi estatisticamente significante $\left(\chi^{2}=5,01 ; p=0,03\right)$, conforme Tabela 1 .

Tabela 1 - Distribuição das pacientes com mastalgia cíclica de diferentes graus de intensidade da dor de acordo com a faixa etária.

\begin{tabular}{|c|c|c|c|c|c|c|c|c|}
\hline \multirow{3}{*}{$\begin{array}{l}\text { Intensidade } \\
\text { da dor }\end{array}$} & \multicolumn{8}{|c|}{ Idade (anos) } \\
\hline & \multicolumn{2}{|c|}{$20-29$} & \multicolumn{2}{|c|}{$30-39$} & \multicolumn{2}{|c|}{$\geq 40$} & \multicolumn{2}{|c|}{ Total } \\
\hline & $\mathrm{n}$ & $\%$ & $\mathrm{n}$ & $\%$ & $\mathrm{n}$ & $\%$ & $\mathbf{n}$ & $\%$ \\
\hline Grau I (leve) & 11 & 22,9 & 21 & 44,8 & 13 & 39,3 & 45 & 35,1 \\
\hline Grau II (moderado) $^{*}$ & 29 & 60,5 & 19 & 40,4 & 18 & 54,7 & 66 & 51,7 \\
\hline Grau III (intenso)* & 8 & 16,6 & 7 & 14,8 & 2 & 6,0 & 17 & 13,2 \\
\hline Total & 48 & 100,0 & 47 & 100,0 & 33 & 100,0 & 128 & 100,0 \\
\hline
\end{tabular}

${ }^{*} \chi^{2}=5,01 ; p=0,03$

A paridade das pacientes está representada na Tabela 2. Observa-se que as nuliparas constituíram o grupo com o maior percentual $(92,9 \%)$ de pacientes com mastalgia moderada (grau II) e intensa (grau III), sendo essa diferença estatisticamente significante quando comparada com aquelas de paridade maior $(\mathrm{p}=0,01)$.

Tabela 2 - Distribuição das pacientes com mastalgia cíclica de diferentes graus de intensidade da dor de acordo com a paridade.

\begin{tabular}{|c|c|c|c|c|c|c|c|c|c|c|}
\hline \multirow{2}{*}{$\begin{array}{l}\text { Intensidade } \\
\text { da dor }\end{array}$} & \multicolumn{10}{|c|}{ Paridade } \\
\hline & $\mathrm{n}$ & $\%$ & $\mathrm{n}$ & $\%$ & $\mathrm{n}$ & $\%$ & $\mathrm{n}$ & $\%$ & $\mathrm{n}$ & $\%$ \\
\hline Grau I (leve) & 1 & 7,1 & 13 & 48,2 & 20 & 34,4 & 11 & 37,9 & 45 & 35,1 \\
\hline Grau II (moderado)* & 12 & 85,8 & 11 & 40,7 & 29 & 50,1 & 14 & 48,4 & 66 & 51,7 \\
\hline Grau III (intenso)* & 1 & 7,1 & 3 & 11,1 & 9 & 15,5 & 4 & 13,7 & 17 & 3,2 \\
\hline
\end{tabular}

${ }^{*}$ Teste exato de Fisher; $p=0,01$

As mastalgias de grau III ocorreram com maior freqüência nas pacientes com menarca igual ou menor que 11 anos e, nesse aspecto, houve diferença estatisticamente significante em relação às outras faixas etárias e aos outros graus de intensidade da dor $\left(\chi^{2}=8,70 ; p=0,003 ; R R=3,57\right.$ (IC 95\%: 1,53-8,30)).

A média de idade das pacientes no momento do seu primeiro parto a termo foi de 24 anos (variação entre 13 e 40). Pareceu-nos relevante que, no grupo de grau III de mastalgia, a maior proporção de pacientes $(81,2 \%)$ tenha tido o primeiro parto a termo em idade mais precoce $(\leq 19$ anos), entretanto não houve diferença estatisti- camente significante entre esse grupo e os demais $\left(\chi^{2}=2,83 ; p=0,09 \mathrm{RR}=2,62\right.$ (IC 95\%: 0,79-8,69), Yates $\left._{\text {corr }} \chi^{2}=1,99 ; \mathrm{p}=0,15\right)$.

A média de amamentação entre os grupos foi de 16 meses (variação de 0 a 96). A Tabela 3 mostra que períodos mais longos de amamentação (>12 meses) não estão necessariamente associados com mastalgia de grau mais leve (grau I). Quando comparadas as pacientes que amamentaram por um período maior que 12 meses com aquelas que o fizeram por um tempo menor, encontrou-se um maior índice de mastalgia graus II e III naquele grupo (82,6 versus 55,3\%), sendo essa diferença estatisticamente significante $(p=0,02)$. 
Tabela 3 - Distribuição das pacientes com mastalgia cíclica de diferentes graus de intensidade da dor, de acordo com a duração do maior período de amamentação de um dos filhos.

\begin{tabular}{|c|c|c|c|c|c|c|c|c|c|c|}
\hline \multirow{3}{*}{$\begin{array}{l}\text { Intensidade } \\
\text { da dor }\end{array}$} & \multicolumn{10}{|c|}{ Período de amamentação (meses) } \\
\hline & \multicolumn{2}{|c|}{$\leq 3$} & \multicolumn{2}{|c|}{$4-6$} & \multicolumn{2}{|c|}{$7-12$} & \multicolumn{2}{|c|}{$>12$} & \multicolumn{2}{|c|}{ Total } \\
\hline & $\mathrm{n}$ & $\%$ & $\mathrm{n}$ & $\%$ & $\mathrm{n}$ & $\%$ & $\mathrm{n}$ & $\%$ & $\mathrm{n}$ & $\%$ \\
\hline Grau I (leve) & 17 & 40,5 & 15 & 53,6 & 6 & 40,0 & 4 & 17,4 & 42 & 39,0 \\
\hline Grau II (moderado)* & 18 & 42,8 & 9 & 32,1 & 8 & 53,4 & 15 & 65,2 & 50 & 46,2 \\
\hline Grau III (intensivo) ${ }^{*}$ & 7 & 16,7 & 4 & 14,3 & 1 & 6,6 & 4 & 17,4 & 16 & 14,8 \\
\hline Total & 42 & 100,0 & 28 & 100,0 & 15 & 100,0 & 23 & 100,0 & $108^{*}$ & 100, \\
\hline
\end{tabular}

${ }^{*} \chi^{2}=5,63 ; p=0,02 ; R R=0,39$ (IC 95\%: 0,15-0,98) Yates=4,59; $p=0,03$

*14 pacientes eram nuliparas e entre as pacientes que pariram, 6 não amamentaram.

Das pacientes que não amamentaram, 85\% tinham mastalgia grau II ou III, contra somente $61,2 \%$ das que amamentaram, mas não houve diferença estatisticamente significante entre esses dois grupos.

A Tabela 4 mostra um resumo dos melhores resultados relativos à eficiência do tratamento (orientação verbal), levando em consideração os fatores estudados no grupo de mulheres com mastalgia cíclica.

Tabela 4 - Resumo dos fatores associados com maior freqüência à eficiência do tratamento não medicamentoso (orientação verbal).

\begin{tabular}{lrcrcc}
\hline Fatores & \multicolumn{2}{c}{$\begin{array}{c}\text { Eficiência do } \\
\text { tratamento }\end{array}$} & Total & $\mathbf{p}$ \\
& $\mathbf{n}$ & $\%$ & $\mathbf{n}$ & $\%$ & \\
\hline Primíparas & 19 & 70,3 & 27 & 100,0 & 0,19 \\
$1^{0}$ parto ( $\geq 30$ anos) & 2 & 66,6 & 3 & 100,0 & 0,80 \\
Idade ( $\geq 40$ anos) & 26 & 78,8 & 33 & 100,0 & 0,01 \\
Menarca (12-14 anos) & 60 & 61,8 & 97 & 100,0 & 0,31 \\
Não amamentaram & 14 & 70,0 & 20 & 100,0 & 0,32 \\
Amamentação ( $\leq 3 m e s e s)$ & 26 & 61,9 & 42 & 100,0 & 0,54 \\
\hline
\end{tabular}

Observou-se que as primíparas, as que tiveram o primeiro parto a termo com idade igual ou superior a 30 anos, as que se encontravam na faixa etária igual ou maior que 40 anos, as que tiveram a menarca entre 12 e 14 anos e as que não amamentaram ou o fizeram por um período de tempo igual ou menor do que três meses responderam melhor ao tratamento proposto.

Por outro lado, só encontramos diferença estatisticamente significante $(p=0,01)$, de fato, no grupo de pacientes com 40 anos ou mais em relação ao grupo de pacientes mais jovens como um todo ( $<40$ anos).

\section{Discussão}

Apesar de não haver consenso na literatura pesquisada em relação ao curso e à prevalência da mastalgia, no que diz respeito à paridade ${ }^{3-5}$, as nuliparas constituíram o grupo com o maior percentual de mastalgia moderada e intensa nas nossas observações. Talvez por essa razão, a melhor resposta à orientação verbal tenha ocorrido nas primiparas, portadoras em maior proporção de mastalgias leves. Contudo, não houve diferença estatisticamente significante em relação a esse aspecto.

Por outro lado, não encontramos na literatura pesquisada referência de como a paridade pudesse de alguma maneira influenciar a resposta ao tratamento não-medicamentoso das mastalgias cíclicas. Esse aspecto é também verdadeiro em relação aos outros fatores estudados, como: idade, menarca, amamentação, etc.

No que diz respeito à idade do primeiro parto a termo, nossas observações sugerem, embora sem significância estatística, quando comparadas aos outros grupos, que as pacientes que tiveram o primeiro parto a termo em idade mais precoce têm maior possibilidade de apresentar mastalgia acentuada (grau III). O risco relativo é de 2,6, segundo os dados obtidos, e corresponde à expectativa da literatura pesquisada ${ }^{6}$. A orientação verbal foi mais eficiente nas pacientes que tiveram o primeiro parto a termo com idade igual ou superior a 30 anos, mas sem diferença estatisticamente significante.

Estudando as pacientes que amamentaram por um período maior ou menor de tempo, não constatamos diferença estatisticamente significante entre os grupos no que diz respeito à resposta ao tratamento não-medicamentoso. Também, não observamos diferença estatisticamente significante entre as pacientes que amamentaram ou não, no que diz respeito à intensidade da dor, apesar de Wetzig ${ }^{2}$ identificar nas mulheres que não amamentaram um grupo com maior prevalência 
de mastalgias. Os resultados obtidos com o tratamento utilizado foi semelhante nos dois grupos estudados.

Uma eficiência maior nos resultados do tratamento foi demonstrada nas pacientes com idade igual ou maior que 40 anos $(78,8 \%)$, com diferença estatisticamente significante quando comparadas com pacientes mais jovens. De fato, as mastalgias de graus II e III (mais intensas) foram mais prevalentes entre as idades de 20 e 29 anos $(77,1 \%)$ contra $60,7 \%$ na faixa etária igual ou acima dos 40 anos), e isso deve ter feito a diferença. Pode-se ainda especular que pacientes nessa faixa etária tenham maior maturidade emocional e se deixem influenciar positivamente pelos aspectos psicológicos em jogo nesse tipo de abordagem. Apesar de não termos tido pacientes com idade abaixo de 20 anos na nossa casuística, é pertinente comentar que o início de dor cíclica na mama, antes da idade de 20 anos, é seguido normalmente por um longo período sintomático ${ }^{1,16}$. Afirmam ainda esses autores que a idade do início da sintomatologia permite predizer, de alguma maneira, o curso da doença. Pacientes jovens com sintomas intensos devem esperar muitos anos de sintomatologia.

A constatação de que a mastalgia intensa (grau III) ocorreu com maior freqüência nas pacientes com menarca igual ou menor que 11 anos do que nas demais pode permitir a associação: ciclo menstrual $\mathrm{x}$ mastalgia cíclica $\mathrm{x}$ imaturidade emocional e/ou nivel de ansiedade maior. O risco relativo de 3,57 , com intervalo de confiança entre 1,53 e 8,30, é altamente significativo. O grupo que respondeu melhor ao tratamento teve menarca entre 12 e 14 anos, mas não houve diferença estatisticamente significante quando o comparamos com os demais. Correspondeu exatamente às pacientes com o menor percentual de mastalgia intensa.

Assim, esperamos que, em face destas considerações, os nossos resultados estimulem mais pesquisas sobre o tema considerando, com um maior número de casos e ampliação de elementos avaliados particularmente, a falta de informações na literatura pesquisada.

\section{SUMMARY}

Purpose: to evaluate factors that might influence the results of nonphamacological treatment (verbal guidance) in women with clinical mastalgia.

Methods: an uncontrolled study of the experimental type was conducted on a sample of 128 women with a clear history of clinical mastalgia treated with verbal guidance. A visual analogue scale for pain was used before and after treatment to evaluate pain intensity and mastalgia was classified into grade I (mild), grade II (moderate) and grade III (severe) according to pain intensity. The Cardiff Breast Score (CBS), modified, was also used to evaluate the clinical response to treatment. Data were analyzed statistically using the $\chi^{2}$ test (Epi-Info 6.04).

Results: we noted that factors such as parity, menarche, age at first term delivery and breast-feeding had no significant effect ( $p=0.19, p=0.31, p=0.80$ and $p=0.54$, respectively) on the results of nonpharmacological treatment (verbal guidance). On the other hand, when age was taken into consideration, 26 patients (78.8\%) aged 40 years or older were found to benefi more from verbal guidance, with a significant difference $(p=0.01)$ when compared to younger women.

Conclusion: reproductive factors such as parity, menarche, age at first term delivery and breast-feeding did not affect the results of nonpharmacological treatment (verbal guidance), whereas the age factor had a specific and significant effect on the results.

KEY WORDS: Breast: benign diseases. Mastalgia. Verbal guidance.

\section{Referências}

1. Wisbey JR, Kumar S, Mansel RE, Peece PE, Pye JK, Hughes LE. Natural history of breast pain. Lancet $1983 ; 2: 672-4$.

2. Wetzig NR. Mastalgia: a 3 year Australian study. Aust N Z J Surg 1994; 64:329-31.

3. Mansel RE. ABC of breast diseases. Breast pain. BMJ 1994; 309:866-9.

4. Barros ACSD, Adamek EV, Muller AP, et al. Perfil epidemiológico da mastalgia em uma população universitária brasileira. Rev Bras Mastol 1995; 5:24-30.

5. Gateley CA, Bundred NJ, West RR, Mansel RE. Reproductive factors associated with mastalgia. Cancer Detect Prev 1992; 16:39-41.

6. Fentiman IS. Mastalgia mostly merits masterly inactivity. Br J Clin Pract 1992; 46:158.

7. Gateley CA. The powerful placebo. Lancet 1991; 337:927.

8. Tavaf-Motamen H, Ader DN, Browne MW, Shriver CD. Clinical evaluation of mastalgia. Arch Surg 1998; 133:211-4.

9. Ader DN, Shriver CD, Browne MW. Cyclical mastalgia: premenstrual syndrome or recurrent pain disorder? J Psychosom Obstet Gynecol 1999; 20:198-202. 
10.Padden DL. Mastalgia: evaluation and management. Nurse Pract Forum 2000; 11:213-8.

11. Sivini FN, Molina A, Costa CFF, Sivini FMP. Mastalgias cíclicas: tratamento nãomedicamentoso (orientação verbal). Rev Bras Ginecol Obstet 2001; 23:77-82.

12.Fentiman IS, Hamed H. Benign breast disease: who and how to treat for mastalgia. In: Figueira ASS, Dias EN, Silva HMS, Barbosa CSD, editors. Mastology - Breast disease. Proceedings of the 8th International Congress on Senology; 1994 May 8-12; Rio de Janeiro, Brasil. Amsterdan: Elsevier; 1995. p.10-1.
13.Steinbrunn BS, Zera RT, Rodriguez JL. Mastalgia. Tailoring treatment to type of breast pain. Postgrad Med 1997; 102:183-98.

14.Abriata G, Bahamonde J, Bazzani M. Tamoxifen therapy for cyclical mastalgia: dose randomized trial. Breast 1997; 5:212-3.

15.Gateley CA, Miers M, Mansel RE, Hughes LE. Drug treatments for mastalgia: 17 years experience in the Cardiff Mastalgia Clinic. J R Soc Med 1992; 85:12-5.

16.Davies EL, Gateley CA, Miers M, Mansel RE. The long-term course of mastalgia. J R Soc Med 1998; 91:462-4.

\section{$5^{\circ}$ Conglesso Mundial de Medicina Perinatal}

\section{3 a 27 de setembro de 2001}

\section{Barcelona - Espanha}

\section{Informação: 3493 238-8777}

fundacio@iudexeus.uab.es 